\title{
1 Modelling total solar irradiance from PMOD composite using feed-forward neural networks
}

\author{
A.Tebabal $^{\mathrm{a}, *}$, B.Damtie $^{\mathrm{a}}$, M.Nigussie $^{\mathrm{a}}$, A. Bires $^{\mathrm{a}}$, E.Yizengaw $^{\mathrm{b}}$ \\ ${ }^{a}$ Washera Geospace and Radar Science Laboratory(WaGRL), Bahir Dar University, \\ Ethiopia \\ ${ }^{b}$ Institute for Scientific Research, Boston College, USA
}

7 Abstract

8 The variability of the solar activity dominates the variability of the earth's at9 mosphere, which affects human life and technology on earth. To understand

\footnotetext{
*Corresponding author

Email addresses: ambelut@bdu.edu.et (A.Tebabal), baylied@bdu.edu.et (B.Damtie), melesssewnigussie@yahoo.com (M.Nigussie), abbi@yahoo.com (A. Bires), kassie@bc.edu (E.Yizengaw)

(C) 2015. This manuscript version is made available under the Elsevier user license http://www.elsevier.com/open-access/userlicense/1.0/
} 
cle 23. Predicting TSI using NNs further strengthens the view that surface magnetism indeed plays a dominant role in modulating solar irradiance.

Keywords: Solar irradiance, sunspot, neural network

\section{Introduction}

Total solar irradiance (TSI), is the total power from the sun impinging on the Earth and its variation in solar radiative output clearly have the potential to affect our planet. This radiative output heats the land and ocean, maintains our atmosphere, generates clouds, and cycles water (Rottman, 2006). Studies conducted by astrophysical and meteorological communities have shown the fingerprint of solar activity on the Earth's climate (Beer et al., 2000; Gleisner \& Thjill, 2003, Scafetta \& West, 2006; Schrijver et al., 2011; Lean et al., 1995). Also researchers are working to understand the physical mechanism responsible for this influence (see,e.g., Ermolli et al., 2013; Krivova \& Solanki, 2013, for a recent review).

For more than three and half decade solar irradiance has been monitored from space by different satellites and from these consecutive and partly overlapping time series measurements three TSI composites have been compiled. These are: the Active Radio Irradiance Monitor (Willson \& Mordvinov, 2003), the Royal Meteorological Institute of Belgium (RMIB) (Dewitte et al., 2004) and the Physikalisch-Meteorologisches Observatorium Davos (PMOD) (Fröhlich, 2006). Each composite has their own calibration techniques and mathematical algorithms. They show certain differences, of which the most important regards the presence of an upward secular trend between the minima preceding cycles 22 (1986) and 23 (1996) (Krivova et al., 2011). 
It is well known that following the 1978 satellite solar irradiance data, various empirical and semi-empirical models have been developed to reconstruct total solar irradiance from solar activity. The models which assume that irradiance variations on a time scale longer than roughly a day are caused their changes in the surface distribution of different magnetic features have turned out to be most successful in explaining observed irradiance changes (Solanki et al., 2013). Authors like Fröhlich \& Lean (2004); Balmaceda et al. (2007); Vaquero et al. (2006) used empirical TSI modelling to address the question of the extent to which variations in TSI can be explained using only variations due to magnetic features such as sunspots, faculae and network. Sunspots cause deficits in the irradiance, while faculae and network cause excesses. The fact that the TSI is on average higher near sunspot maximum implies that the influence of faculae and network is greater than that of sunspots (Fröhlich \& Lean, 1998). Proxy-based models (see, Ashamari et al., 2015, for a recent review) try to answer the question: to what extent specific solar proxies, such as sunspot number, Mg II index, F10.7 radio flux, abundance of cosmogenic isotopes, spectral lines or specific wavelength bands in the observed solar spectra or solar intensitygrams, can be used to describe solar irradiance variability. This proxy-based empirical model is very successful in reproducing the observed TSI variations from 1978 to 2004, but it fails to explain the decrease observed since 2005 (Fröhlich, 2009). The advantage of employing radiances computed from the semi-empirical model atmospheres is that they allow computations of solar irradiance at different wavelength (i.e. spectral irradiance) (Yeo et al. 2014a b). This is not straightforward with the regression models, since in this case the regression coefficients need 
to be estimated from observations (Ermolli et al., 2013). Modelling of TSI is of both scientific and practical interest.

Methods of artificial intelligence have provided tools which potentially make the task of solar irradiance modelling and forecasting possible. For instance, previous works (see, Mellit \& Pavan, 2010; Alzahrani \& Kimball, 2014; Faceira et al., 2015, for a detail review) showed that the feasibility of the neural networks approach in modelling the non-linear relationship between solar radiation and geographical and meteorological parameters. Results of their study showed that neural networks allow estimating the values of radiation with great precision in various regions of the world. However, in this paper we concentrate on TSI modelling from PMD composite that was compiled from different space-borne measurements using feed-forward neural networks approach.

The main goal of this paper is to investigate whether the feed-forward neural networks (NNs) model can explain the recently observed negative trend in the TSI, or an additional component as proposed in Fröhlich (2009) is needed. For this purpose, two most commonly used basic indices : the photometric Sunspot Index (PSI) and Mg II index were used as a proxy data for solar magnetic activity. As pointed out by Fröhlich \& Lean (2004) the Mg II index is treated into the long- and short-term components, $\mathrm{Mg} \mathrm{II}_{l t}$ and $\mathrm{Mg}$ $\mathrm{II}_{s t}$, respectively. The long-term component was calculated by convolving the Mg II index with an 81-day Full-Width Half-Maximum (FWHM) Gaussian function. The difference between Mg II index and long-term component represents the short-term component. Our investigation is carried out for the time interval of 1978 to 2013, which represents nearly four solar cycles. 


\section{The measurement and analysis}

\subsection{The measurement}

The input data have been taken from the solar indices that have impact on the TSI variations such as the daily PSI, and composite Mg II index. The daily PSI indicates the solar activity and describes the radiation deficit caused by the spots. Sunspots can be modelled from their area and position on the disk by using an appropriate contrast and its result is PSI, which is given by part per million (ppm) of TSI. Daily PSI has been compiled by Balmaceda et al. (2009) and is available at: http://www2. mps.mpg.de/projects/sunclimate/data/table4_v0613.txt. This data is available starting from 1874, however, PSI data after November 17, 1978 to 2013 has been considered for our study. The composite Mg II index from 1978 to 2013, which is available at http://www.iup.uni-bremen.de/gome/ gomemgii.html, was used to estimate the effect of faculae and network on solar irradiance Variability. This Mg II index, which is the core-to-wing ratio of the irradiance in the core of the unresolved Mg II doublet at 280 $\mathrm{nm}$ to the nearby continuum irradiance and measures solar variability on both rotational and solar cycle time scales (Deland \& Cebula, 1993). Thus, these indices are considered as an input parameter for TSI modelling by considering their short term as well as long term influences on solar activity. Total solar irradiance data version d41_62_1302 from PMOD/WRC, Davos, Switzerland, used as a target. Figure 1 shows daily values (black) and 191day running means (red) and from top to bottom: the PMOD composite of the TSI, PSI, Mg II $s t$, and $\mathrm{Mg} \mathrm{II}_{l t}$ from the year 1978-2013 respectively. 


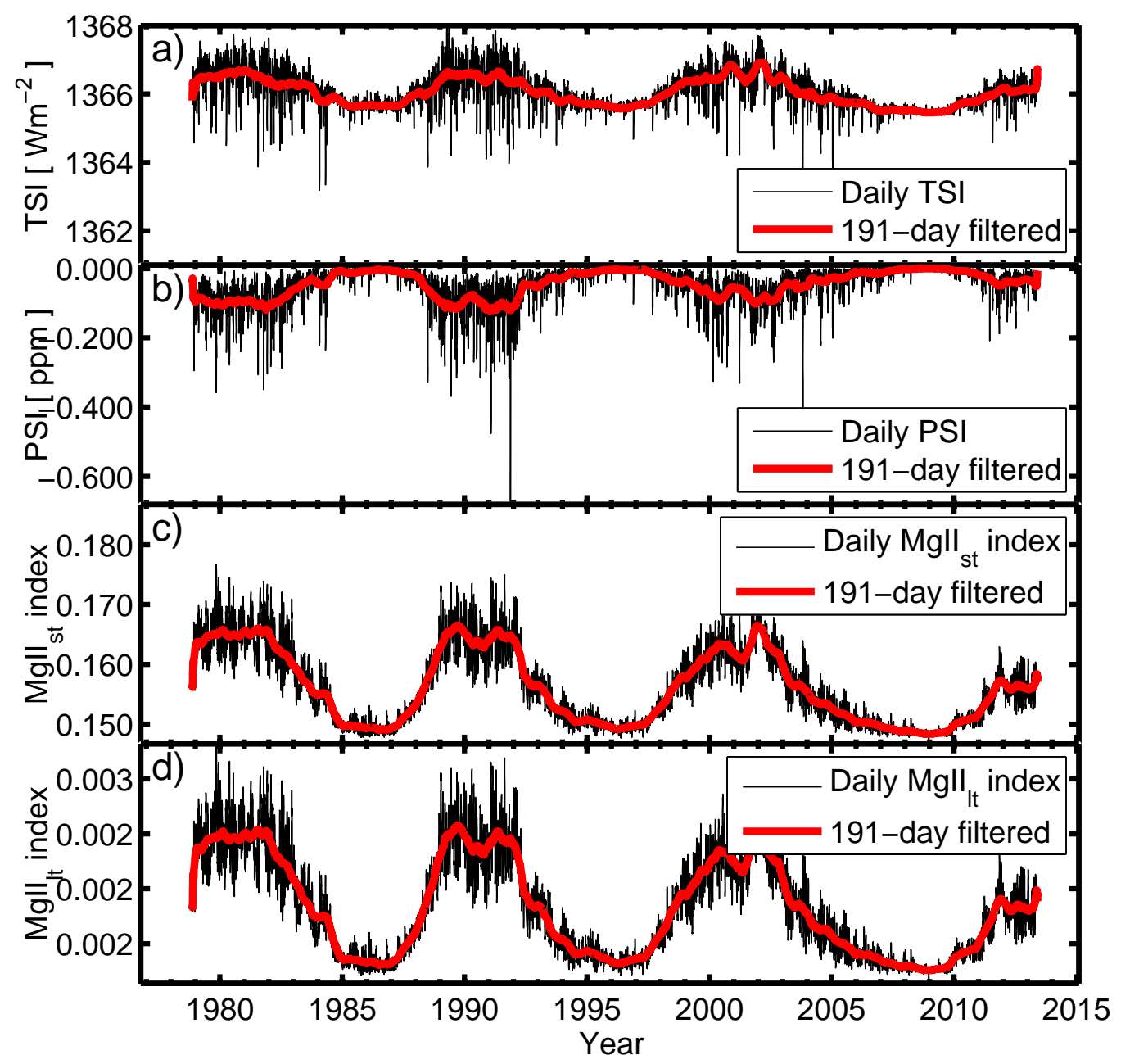

Figure 1: Daily values of the time series a) TSI (PMOD composite) b) photometric sunspot index (PSI) c) Mg II $s t$ and d) $\mathrm{Mg} \mathrm{II}_{l t}$ index. The period covers from November 17, 1978 up to October 31, 2013. 


\subsection{The analysis}

Neural networks $(\mathrm{NNs})$ are mathematical system which can be able to model the ability of biological neural networks by interconnecting many simple neurons. The neuron accepts inputs from a single or multiple sources and produces an output by training process with a predetermined non-linear function. NNs are capable of directly correlating the time series of multiple input variables to the output variables through the interconnected nodes using trainable weights and bias signal (Hagan et al. 1995). NN is an important tool for non-linear approximation when it is trained with sufficient historic data (Habarulema et al., 2007).

The two primary components of the NNs are the processing elements and their interconnections. Figure 2 shows a fully connected single hidden layer feed-forward NNs. As it is shown in the figure, there are three categories of layers in a neural network: input, hidden and output layers. Each layers is made up of several nodes, and layers are interconnected by sets of correlation weights. And also, each layer's computation is split into two components. First, a linear component, called the input function, computes the weighted sum of the layer's input values. For instance, in the case of seven hidden neuron $\mathrm{j}$, we have:

$$
\text { net }_{j}=\sum_{i=1}^{3} w_{j i} x_{i}+b_{j},
$$

where $\mathrm{x}_{i}$ represents the input variables (such as PSI, $\mathrm{Mg} \mathrm{II}_{l t}$, and $\mathrm{Mg} \mathrm{II}_{s t}$ ), $\mathrm{w}_{i j}$ is the weights of the neuron $\mathrm{j}$, net ${ }_{j}$ is the linear combiner output due to the input signals, and $\mathrm{b}_{j}$ is the bias of the hidden neurons. Second, a nonlinear component, called the activation function, transforms the weighted sum $\left(\right.$ net $\left._{j}\right)$ into layer's final activation value. Different kinds of functions 


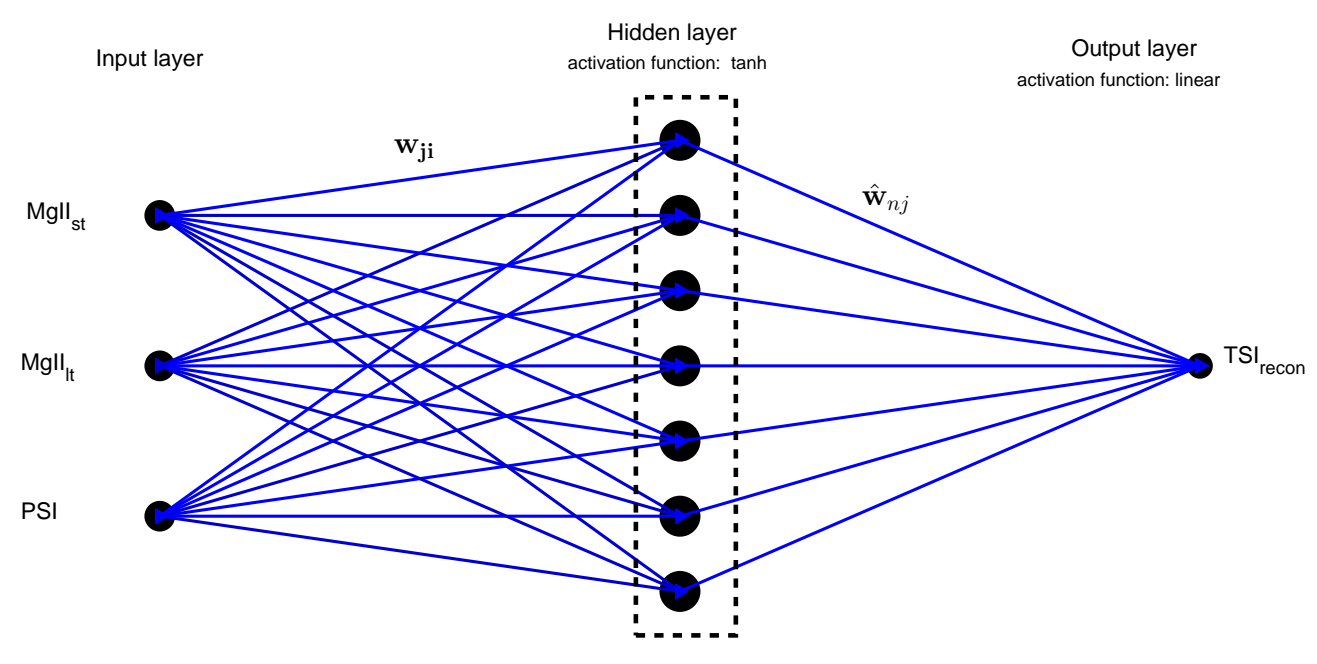

Figure 2: Schematic of a single hidden layer, feed-forward neural network to reconstruct TSI.

$$
f\left(\text { net }_{j}\right)=1-\frac{2}{\left(\exp \left(2 n e t_{j}\right)+1\right)}
$$

Then, the outputs of all the hidden neurons are weighted and sent to the output layer, which contains a unique node n. It returns the prediction as:

$$
T S I_{\text {pre }}=\sum_{j=1}^{7} \hat{w}_{n j} f\left(\text { net }_{j}\right)+\hat{b}_{j}=\sum_{j=1}^{7} \hat{w}_{n j} f\left(\sum_{j=1}^{3} w_{j i} x_{i}+b_{j}\right)+\hat{b}_{n j} .
$$

where $\mathrm{TSI}_{\text {pre }}$ is the predicted TSI of the output neuron, $\hat{w}_{j}$ is the weight of 
the output of the $j^{\text {th }}$ hidden neuron at the output neuron, and $\hat{b}_{j}$ represents the bias associated to the output neuron.

Learning occurs through the adjustment of the weights and node biases. The most common method used for adjustment is back-propagation. Back-propagation can train multilayer feed-forward networks with differentiable transfer functions to perform function approximation, pattern association, and pattern classification (Demuth \& Beale, 2000). The term backpropagation refers to the process by which derivatives of network error, with respect to network weights and biases, can be computed.

The error at the output layer in the back-propagation NNs model propagates backward to the input layer through the hidden layer in the network to obtain the final desired output. This process can be used with a number of different optimization strategies. The detail analysis of back-propagation algorithm is given by Gong et al. (2012); Haykin (1999); Svozil et al. (1997); Rumelhart et al. (1986) and Bishop (1993). This means the desired behavior can be evaluated by the error function E, which is given by:

$$
E=\frac{1}{2} \sum_{j=1} E_{j}^{2}=\frac{1}{2} \sum_{j=1}\left(T S I_{\text {meas }}^{j}-T S I_{\text {pre }}^{j}\right)^{2} .
$$

where $\mathrm{TSI}_{\text {meas }}$ and $\mathrm{TSI}_{\text {pre }}$ represent the target and predicted values of output neuron, respectively.

\subsection{Neural network training and performance measures}

Among the various NNs structures, we have used a basic structure known as a feed-forward neural network with Levenberg-Marquardt (LM) backpropagation algorithm. The LM algorithm was designed to approach secondorder training speed without having to compute the Hessian matrix. This 
method combines, in the same weight updating rule, both the gradient and the Gauss-Newton approximation of the Hessian of the error function. This makes it less complicated and memory demanding, that is why it is often used in NNs training and was chosen also for the purpose of this study. A more detailed description of LM algorithm can be found in Mellit \& Pavan (2010).

The network is feed-forward because the elements in one layer are only connected to the elements in the next layer and the information moves forward from one layer to another toward the output layer. At the training stage, both the inputs and the desired output (TSI) are presented to the network. Through learning algorithm the network produces its own output and tries to minimize the discrepancies between its own output and the measured TSI. After training and verification have been successfully completed, the model weight and bias parameters can be saved for future application. We applied batch training process, in which weights and biases are only updated after all the inputs and targets are presented.

A well-known problem with NNs models like feed-forward is that they can overfit the training data and not generalize well to new data outside the training set (Srivastava et al., 2014; Soński, 2011; Demuth \& Beale, 2000). This can be prevented by using various methods like early stopping, prunning, Bayesian regularization and weight decay to minimize the risk of over-fitting. In the case of early stopping technique improvement of generalization of the training can be achieved by dividing the available data into three subsets. Here we follow the same procedure by dividing the data into training, validation and testing set. Data from November 17, 1978 to September 25, 2003 
with 8151 observations are used for model building, and the next 1746 observations in September 26, 2003 and July 25, 2008 are used for the validation purpose. The out-of-sample period is from July 26, 2008 to October 31, 2013 and consists of the last 1746 data points which are used for testing. The model building data set is used to estimate model parameters and validation set is to check the performance of the network to determine the epoch at which training should be stopped to avoid over-fitting or over-training. The testing set is used to assess final overall performance of the NNs.

In order to achieve optimum NNs model, Root-Mean-Square Error (RMSE), correlation coefficient and the absolute error are used in the analysis. The RMSE is also known as the fit standard error and the standard error of the regression. It is an estimate of the standard deviation of the random component in the data, and is defined by:

$$
R M S E=\sqrt{\frac{1}{N} \sum_{i=1}^{N}\left(T S I_{\text {meas }}^{i}-T S I_{p r e}^{i}\right)^{2}}
$$

where $\mathrm{N}$ is the number of data points, $T S I_{\text {meas }}$ is the measured data by using satellites, and $T S I_{\text {pre }}$ is predicted by the model (Härdle \& Simar, 2003). In addition to RMSE, the model efficiency $\mathrm{R}^{2}$ and mean absolute error were used as an indictor of the goodness of fit in this study. The model efficiency can be measured by:

$$
R^{2}=1-\frac{\sum_{i=1}^{N}\left(T S I_{\text {meas }}^{i}-T S I_{\text {pre }}^{i}\right)^{2}}{\sum_{i=1}^{N}\left(T S I_{\text {meas }}^{i}-T S I_{\text {mean }}\right)^{2}} .
$$

The mean absolute error refers to Eq. 7 and can be expressed by

$$
M A E=\frac{1}{N} \sum_{i=1}^{N}\left|T S I_{\text {meas }}^{i}-T S I_{\text {pre }}^{i}\right| .
$$




\section{Results and discussion}

A number of training runs have been performed using different numbers of hidden neurons, and the statistics of the model performance for training, validation and testing set are summarized in Table 1. As shown in this table the network with single hidden layer of 7 hidden neurons has achieved the best performance. The computed correlation coefficient value $(\mathrm{R})$ between the NNs output and the PMOD composite reflects the accuracy of the training process. It is known that NNs interpolate well within the in-sample data than for the out-of-sample period, and therefore the network is expected to reproduce the data set that was used to train it with relatively good accuracy (Habarulema et al., 2007; Habarulema \& McKinnell, 2012). For this purpose, the generalisation capability of the considered feed-forward NNs model is assessed in prediction out-of-sample daily TSI data from July 26, 2008 to October 31, 2013. For prediction (extrapolation) period the computed RMSE and MAE were 0.1274 and $0.0823 \mathrm{Wm}^{-2}$ respectively and these results show that NN-predicted TSI values are very close to the testing set. The achievement of the trained network depends not only on the input parameters and the number of hidden neurons but also on the initial weights and biases. Therefore, initial weights and biases for the training process are set to be random values.

The temporal comparison of model prediction and PMOD composite during training, validation and testing phases are given in Figure 3 a from the year 1978 to 2013. Figure 3b shows the residuals between PMOD composite and NNs model output. This residual is almost flat in all considered cycles. The bottom panel of Figure 3c shows the overall trend in the PMOD com- 
Table 1: Results of NNs techniques for training, validation and testing data set.

\begin{tabular}{c|cccccc}
\hline \multirow{2}{*}{$\begin{array}{c}\text { No of } \\
\text { hidden }\end{array}$} & \multicolumn{2}{c|}{ Training } & \multicolumn{2}{c|}{ Validation } & \multicolumn{2}{c}{ Testing } \\
neuron & $\begin{array}{c}\text { RMSE (MAE) } \\
\left(\mathrm{Wm}^{-2}\right)\end{array}$ & $\mathrm{R}$ & $\begin{array}{c}\text { RMSE(MAE) } \\
\left(\mathrm{Wm}^{-2}\right)\end{array}$ & $\mathrm{R}$ & $\begin{array}{c}\text { RMSE(MAE) } \\
\left(\mathrm{Wm}^{-2}\right)\end{array}$ & $\mathrm{R}$ \\
\hline 1 & $0.2301(0.1576)$ & 0.9174 & $0.1190(0.0936)$ & 0.9533 & $0.1421(0.1075)$ & 0.9360 \\
2 & $0.2203(0.1260)$ & 0.9243 & $0.1482(0.1016)$ & 0.9334 & $0.1467(0.1128)$ & 0.9340 \\
3 & $0.2270(0.1570)$ & 0.9194 & $0.1178(0.0836)$ & 0.9486 & $0.1439(0.1062)$ & 0.9424 \\
4 & $0.2178(0.1521)$ & 0.9261 & $0.1375(0.0975)$ & 0.9407 & $0.1467(0.1116)$ & 0.9347 \\
5 & $0.2237(0.1569)$ & 0.9218 & $0.1459(0.0876)$ & 0.9325 & $0.1331(0.0992)$ & 0.9493 \\
6 & $0.2215(0.1529)$ & 0.9235 & $0.1389(0.1025)$ & 0.9480 & $0.1439(0.1113)$ & 0.9364 \\
7 & $0.2271(0.1586)$ & 0.9200 & $0.1316(0.0855)$ & 0.9389 & $0.1274(0.0823)$ & 0.9394 \\
8 & $0.2387(0.1627)$ & 0.9106 & $0.1251(0.0955)$ & 0.9434 & $0.1542(0.1100)$ & 0.9219 \\
9 & $0.2420(0.1621)$ & 0.9081 & $0.1267(0.1003)$ & 0.9457 & $0.1512(0.1139)$ & 0.9312 \\
10 & $0.2261(0.1566)$ & 0.9201 & $0.1620(0.0918)$ & 0.9067 & $0.1376(0.1043)$ & 0.9452 \\
11 & $0.2292(0.1567)$ & 0.9178 & $0.1885(0.0968)$ & 0.9448 & $0.1467(0.1079)$ & 0.9301 \\
12 & $0.2174(0.1521)$ & 0.9264 & $0.1440(0.0947)$ & 0.9340 & $0.1422(0.1073)$ & 0.93861 \\
\hline
\end{tabular}

posite is well replicated in Spectral And Total Irradiance REconstructions for the Satellite era (SATIRE-S) and NNs modelling approach. The SATIRE-S reconstruction is higher between 1984 and 1994 (see Yeo et al., 2014b, for details) while the NNs prediction between 1984 and 1994 slightly lower by about 0.2 and $0.3 \mathrm{Wm}^{-2}$ from PMOD composite.

To examine the linear association between the total solar irradiance for the training, validation and testing data and NNs model output, we present a scatter plot for each category in Figure 4. The green colored solid line represents the best fit linear regression line between between the NNs prediction and PMOD data sets. In each panel, the corresponding correlation coefficient $\mathrm{R}$, and the linear equations are given. The right bottom panel of 


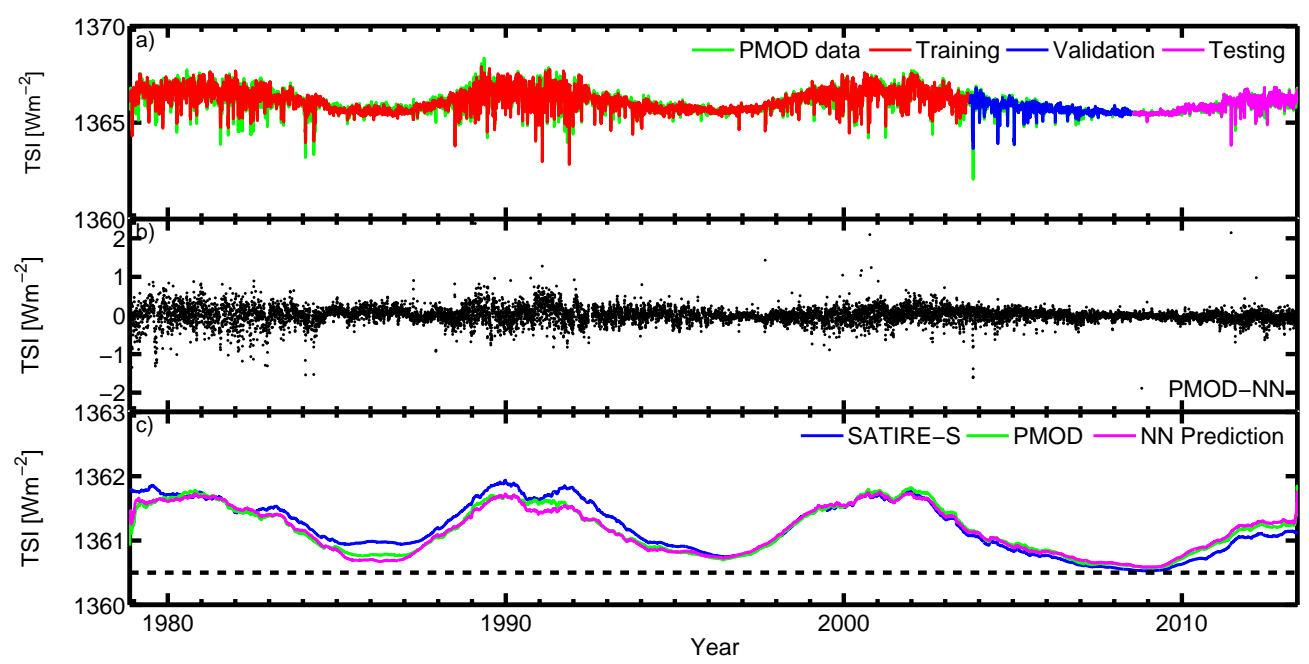

Figure 3: a) NNs reconstruction of TSI (red, blue and magenta), and PMOD composite records (black). b) Difference between NNs predicted TSI, and PMOD composite. c) SATIRE-S (Yeo et al. 2014b reconstruction of TSI, NNs prediction of TSI and PMOD composite records of TSI. All the time series were normalized to TIM at the 2008 solar cycle minimum (horizontal broken line) and smoothed with a 181-day moving average.

the figure shows the scatter plot of the daily TSI values for year 1978-2013 predicted by feed-forward NNs method using data of PMOD composite times series, as a function of PSI and MgII index data. The result shows that the feed-forward NNs model was satisfactorily trained to determine the weight and bias parameters in the network so that the model output well match with the PMOD composite. This performance is achieved based on 11643 data points. The measured TSI and model prediction has a correlation coefficient of $\mathrm{R}=0.9307$, and this means that $86.6 \%$ the total variation of the TSI is 

individual solar cycle including the number of data points is given in Table 2.

Table 2: Statistics of NNs model and PMOD composite time series data correlation, RMSE and regression coefficient for solar cycle 21, 22, 23, and 24 .

\begin{tabular}{l|c|c|c|c|c|c}
\hline Year & Data set & Solar Cycle & $\begin{array}{c}\text { RMSE } \\
\left(\mathrm{Wm}^{-2}\right)\end{array}$ & $\begin{array}{c}\text { MAE } \\
\left(\mathrm{Wm}^{-2}\right)\end{array}$ & $\mathrm{R}$ & $\mathrm{R}^{2}$ \\
\hline $1978-1986$ & 2334 & 21 (part) & 0.2675 & 0.1917 & 0.8993 & 0.7273 \\
$1987-1996$ & 3445 & 22 & 0.2218 & 0.1535 & 0.9219 & 0.8499 \\
$1997-2008$ & 4277 & 23 & 0.1636 & 0.1105 & 0.9565 & 0.9149 \\
$2009-2013$ & 1587 & 24 (part) & 0.1324 & 0.0866 & 0.9327 & 0.8699 \\
\hline
\end{tabular}

280 prediction than to the goodness-of-fit achieved by other empirical models of TSI which is done by Fröhlich \& Lean (2004) using multiple linear regression analysis and their three component model was able to explain $78.6 \%$ of the variance of TSI. The prediction confirms the assumption that great part of the solar irradiance are due to the surface magnetism (sunspot, faculae and networks) on the solar cycle and secular time scales (Krivova et al., 2007; Solanki et al., 2005).

A detailed analysis of the composite time series is presented in Figure 5 and we showed the results of NNs model to PMOD composite time series data for solar cycles 21, 22, 23 and 24 separately. The green dotted point indicates a 27-day running mean of the daily values of TSI obtained using the NNs model, while the dark line shows the 27-day running mean variation in the PMOD composite time series values. The goodness of fit of statistics for

In our analysis a high correlation is found for solar cycle 23. Research findings

due to these solar proxies. This three component NNs model showed a better 

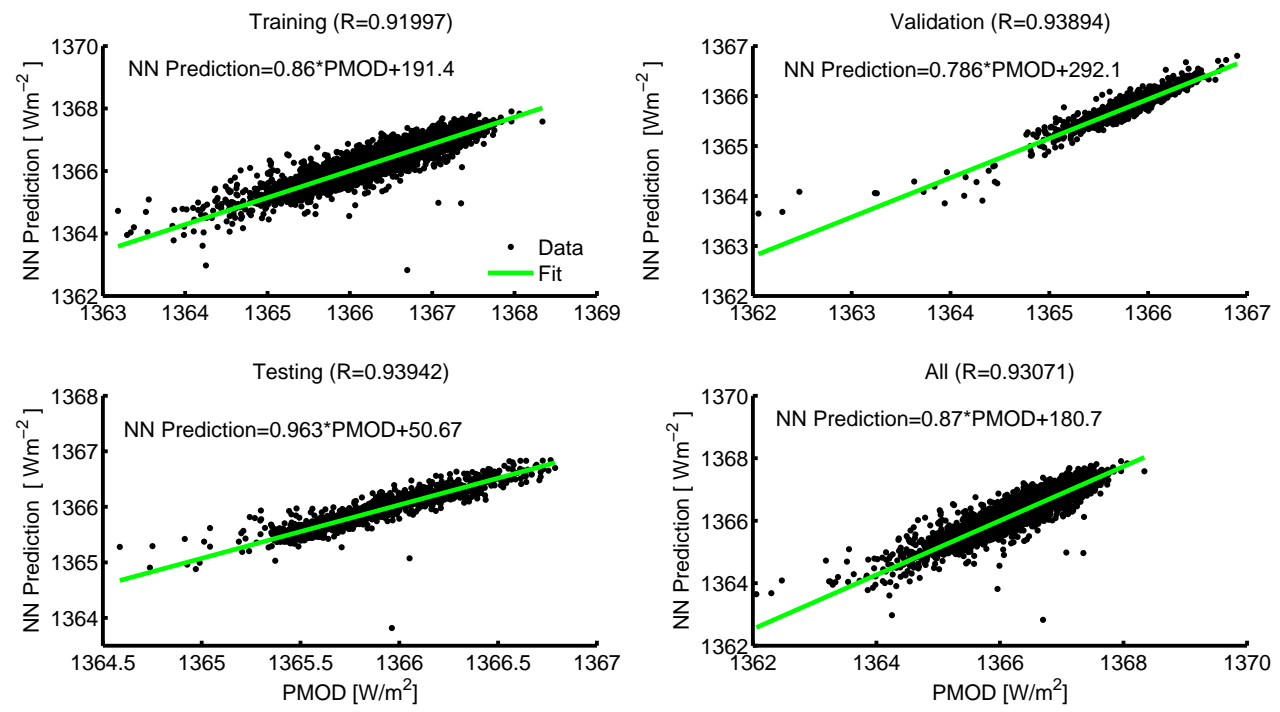

Figure 4: Comparison of the NNs prediction and targets for training, validation and testing data sets.

indicted that at the end of the declining phase for cycle 23 and the beginning of solar cycle 24, the sunspot area was comparatively smaller and Mg II index higher. Therefore, inter-cycle variation can be found between the indices and total solar irradiance (Fröhlich, 2009). The growth of sunspot area is believed to cause changes in the TSI (de Toma et al., 2001), but observations show that the strength of the TSI cycle did not change significantly, despite the decrease in sunspot activity in cycle 23 relative to cycle 22 (de Toma et al. 2004; Fröhlich, 2013). The reconstructed TSI from three component model (Fröhlich, 2009) by using multiple regression doesn't show the pattern of the measured TSI. However, the result of NNs model shows that the predicted TSI using magnetic feature data is well estimated and the pattern of the measured TSI explicitly shown for each solar cycle. The reason is that we 

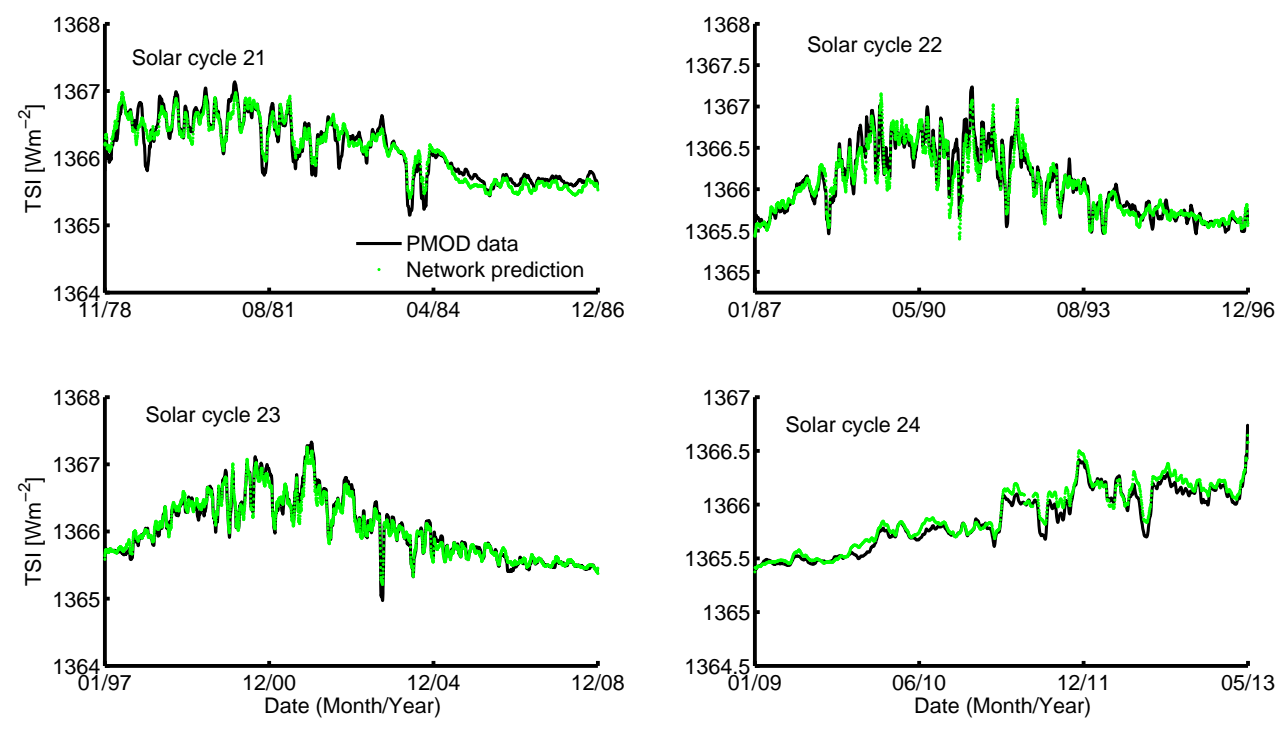

Figure 5: The 27-day running mean of the predicted total solar irradiance using NNs models based on PSI and Mg II index data for solar cycle 21, 22, 23, and 24 is shown by a green dotted line. The 27-day running mean of the observed TSI is shown by the balck solid line.

have used a data driven approach called feed-forward NNs that learns from its error iteratively whereas traditional regression model represents only linear relationships between dependent and independent variables. This allows us to suggest the reason why we cannot explain the pattern of the measured TSI using the model of magnetic features may be due to the model (multiple linear regression) itself. Multiple linear regression model depends on the time resolution and choice of the training period and this is the main shortcoming of this model (see Usoskin, 2013, for a recent review). The pattern of the reconstructed output (TSI) of the model indicates that the solar proxy (PSI and Mg II index) is greatly account for the variance of TSI. 

considered solar cycle 23 periods.
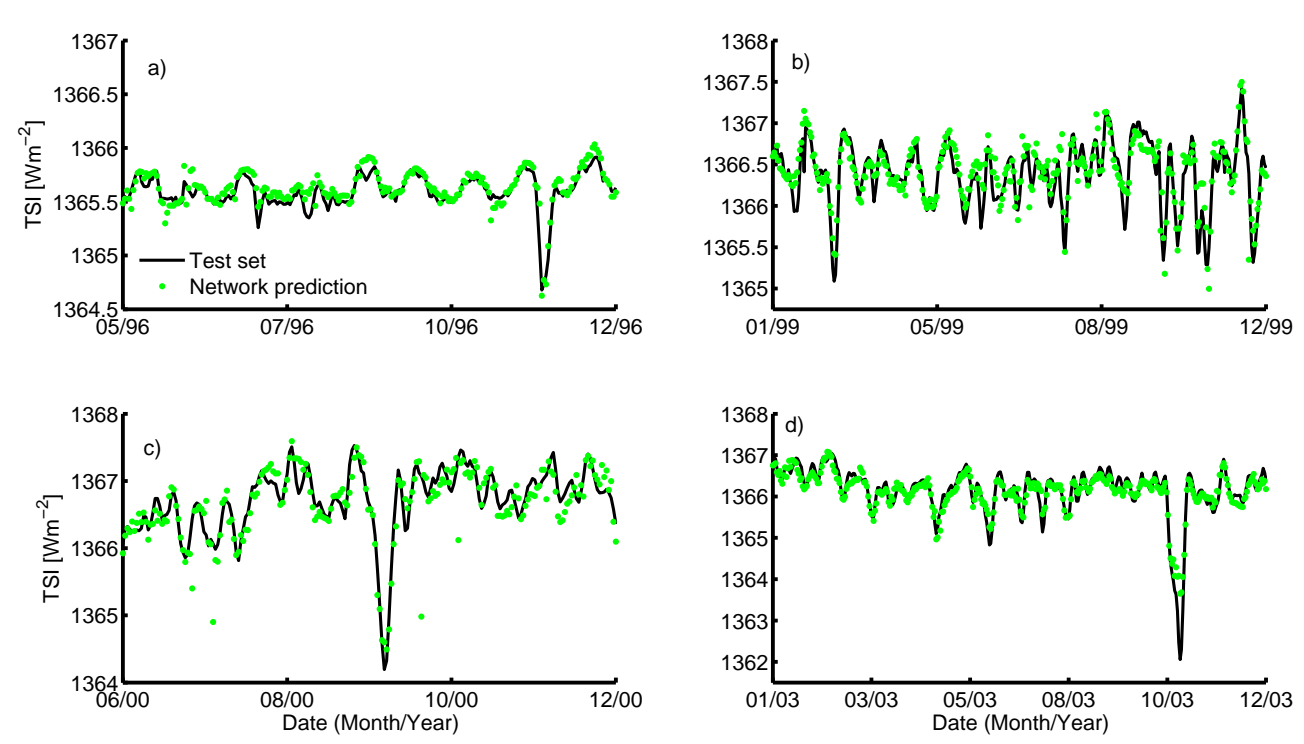

irradiance data with NNs model for solar cycle 23 (Figure 6). We consider the daily variation in the modelled and measured total solar irradiance at low solar activity, rising solar activity, high solar activity, and declining solar activity. The dark solid line is for the TSI data from PMOD composite times series and the dotted (green) are for modeled values. We can see from Figure 6 that the NNs model and the measurement agree well for the

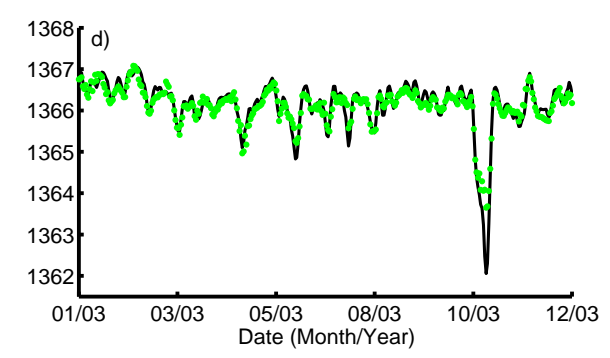

Figure 6: The daily variation in the modelled and measured total solar irradiance at a) low solar activity, b) rising solar activity, c) high solar activity, and d) declining solar activity during cycle 23. The solid line is for the TSI data from PMOD composite times series and the dotted (green) are for modelled values. 


\section{Conclusions and future works}

In this paper, we present the prediction of daily TSI from solar proxies (PSI, Mg $\mathrm{II}_{s t}$ and $\mathrm{Mg} \mathrm{II} l t$ ) by using NNs modelling technique. A single layer feed-forward approach with LM back-propagation algorithm implemented to determine weights and biases in the training. Although the parameters of the model were chosen by experimentation.

The prediction capability of the NNs was evaluated by the statistics of the root mean square error, mean absolute error and the correlation coefficient between PMOD composite and the predicted TSI. A comparison between the PMOD composite and the one predicted using the developed feed-forward network prediction shows a good performance for the out-of-sample data and the their correlation coefficient is about 0.9394. Although, we found a good agreement between PMOD composite and the predicted TSI $(\mathrm{R}=0.9307$ over the span of the record, 1978 to 2013). Therefore, this model has able to explain $86.6 \%$ of the variance of TSI for the entire period. The dynamic nonlinear trend in the PMOD composite is well reproduced in all solar cycles (21-24) except between 1984 and 1994 where the prediction is lower by about 0.2 and $0.3 \mathrm{Wm}^{-2}$ from PMOD composite. This is a substantial improvement to the traditional methods (Fröhlich \& Lean, 2004) which were able to explain only $78.6 \%$ of TSI variations. This improvement is archived due to ability of NNs to represent both linear and non-linear relationships, as compared with traditional regression models which work for linear relationships.

The implication of the close correlation between PMOD composite and the predicted TSI is that the assumption behind the model is good and the surface magnetic field causes the irradiance variations on the time scales of 
days up to the solar cycle. Also we can conclude that using PSI, Mg $\mathrm{II}_{l t}$ and $\mathrm{Mg} \mathrm{II}_{s t}$ as proxy to reproduce the solar irradiance by means of NNs model is useful to understand the temporal variations of the emitted energy at all wavelengths. Our future research work is to extend TSI prediction for epoches before irradiance observation began.

\section{Acknowledgements}

A. Tebabal, M. Nigussie and A. Bires's work has been supported by the grant from AFOSR (FA8655-13-1-3052). We gratefully acknowledge this support. The authors thank receipt of the data set (version d41_62_1302 ) from PMOD/WRC, Davos, Switzerland, PSI data from IMPRS and composite Mg II Index from University of Bremen.

\section{References}

Alzahrani, A., \& Kimball, C., J.W.and Dagli (2014). Predicting solar irradiance using time series neural networks. Procedia Computer Science, 36 , 623-628.

Ashamari, O., Qahwaji, R., Ipson, S., Sch'oll, M., Nibouche, O., \& Haberreiter, M. (2015). Identification of photospheric activity features from soho/mdi data using the asap tool. astro-ph.SR, .

Balmaceda, L., Krivova, N., \& Solanki, S. (2007). Reconstruction of solar irradiance using the group sunspot number. Advances in Space Research, 40, 986-989. 
Balmaceda, L. A., Solanki, S. K., \& Krivova, N. A. (2009). A homogeneous sunspot areas database covering more than 130 years. J. Geophys. Res., 114.

Beer, J., W.Mende, \& Stellmacher, R. (2000). The role of the sun in our climate forcing. Quaternary Science Reviews, 19, 403-415.

Bishop, C. B. (1993). Curvature-driven smoothing: A learing algorithm for feedforward networks. IEEE Transactions on Neural Networks, 4 .

Deland, M. T., \& Cebula, R. P. (1993). Composite mg ii solar activity index for solar cycles 21 and 22. J. Geophys. Res., 98, 12,809-12,823.

Demuth, H., \& Beale, M. (2000). Neural Network Toolbox: For Use with MATLAB. The MathWorks, Inc.

Dewitte, S., Crommelynck, D., Mekaoui, S., \& A.Joukoff (2004). Measurement and uncertaininty of the long-term total solar irradiance trend. Journal of the Royal Statistical Society, Series B, 39.

Ermolli, I., Matthes, K., de Wit, T. D., Krivova, N. A., Tourpali, K., M.Weber, Unruh, Y. C., Gray, L., Langematz, U., Pilewskie, P., Rozanov, E., Schmutz, W., Shapiro, A., Solanki, S. K., \& Woods, T. N. (2013). Recent variability of the solar spectral irradiance and its impact on climate modelling. Atmos. Chem. Phys., 13, 3945-3977.

Faceira, J., Afonso, P., \& Salgado, P. (2015). Prediction of solar radiation using artificial neural networks. In G. V. Antnio Paulo Moreira, Anbal Matos (Ed.), CONTROLO2014-Proceedings of the 11th Portuguese Conference on Automatic Control (pp. 397-406). Springer volume 321. 
Fröhlich, C. (2006). Solar irradince varaiblility since 1978. revision of pmod composite during solar cycle 21. Space Science Reviews, 125, 53-65.

Fröhlich, C. (2009). Evidence of a long-term trend in total solar irradiance. A\&A, 501, L27-L30.

Fröhlich, C. (2013). Total solar irradiance: What have we learned from the last three cycles and the recent minima? Space Sci. Rev., 176, 237-252.

Fröhlich, C., \& Lean, J. (1998). The sun's total irradiance: cycles, trends and climate change uncertainties since 1976. Geophys. Res. Lett., 25, 43774380.

Fröhlich, C., \& Lean, J. (2004). Solar radiative output and its variability: Evidence and mechanisms. The Astronomy and Astrophysics Review, 12, $273-320$.

Gleisner, H., \& Thjill, P. (2003). Patterns of tropospheric response to solar variability. Geophysical Research Letters, 30, 1711.

Gong, L., Liu, C., Li, Y., \& Yuan, F. (2012). Training feed-forward neural networks using the gradient descent method with the optimal stepsize. Journal of Computational Information Systems, 8, 1359-1371.

Habarulema, J. B., McKinnel, L. A., \& Cillers, P. J. (2007). Prediction of global positioning system total electron content using neural networks over south africa. Journal of Atmospheric and Solar Terrestrial physics, $69,1842-1850$. 
Habarulema, J. B., \& McKinnell, L. A. (2012). Investigating the performance of neural network backpropagation algorithms for tec estimations using south african gps data. Ann. Geophys., 30, 857-866.

Hagan, M., Demuth, H., \& Beale, M. (1995). Neural Network Design. PWS Publishing Company, Boston, MA.

Härdle, W., \& Simar, L. (2003). Applied Multivariate Statistical Analysis. ISBN 978-3-642-17228-1. Springer-Verlag Berlin Heidelberg.

Haykin, S. (1999). Neural Networks: A Comprehensive Foundation. PrenticeHall,Inc.

Krivova, N., Balmaceda, L., \& Solanki, S. (2007). Reconstruction of solar total irradiance since 1700 from the surface magnetic flux. Astron. Astrophys., 467, 335346 .

Krivova, N. A., \& Solanki, S. K. (2013). Models of solar total and spectral irradiance variability of relevance for climate studies. Climate and Weather of the Sun-Earth System (CAWSES), Springer Atmospheric Sciences, .

Krivova, N. A., Solanki, S. K., \& Unruh, Y. C. (2011). Twards a long-term record of solar total and spectral irradiance. Journal of Atmospheric and Solar-Terrestrial Physics, 73, 223-234.

Lean, J., Beer, J., \& Bardely, R. (1995). Reconstruction of solar irradiance since 1610: Implications for climate change. Geophysical Research Letters, 22, 3195-3198. 
Mellit, A., \& Pavan, M., A (2010). A 24-h forecast of solar irradiance using artificial neural network: Application for performance prediction of a gridconnected pv plant at trieste, italy. Solar Energy, 84, 807-821.

Norgaard, M., Ravn, M., \& Hansen, O. (2000). Neural Networks for Modelling and Control of Dynamic Systems. Sparing-verlang London.

Rottman, G. (2006). Measurement of total and spectral solar irradiance. Space Science Reviews, 125, 39-51.

Rumelhart, D. E., Hinton, G. E., \& Williams, R. (1986). Learining representations by back-propagating errors. Nature, 323.

Scafetta, N., \& West, B. J. (2006). Phenomenological solar signature in 400 years of reconstructed northern hemisphere temperature record. Geophysical Research Letters, 33.

Schrijver, C. J., Livingston, W. C., Woods, T. N., \& Mewaldt, R. A. (2011). The minimal solar activity in 2008-2009 and its implications for long -term climate modeling. Geophysical Research Letters, 38.

Solanki, S., Krivova, N., \& Wenzler, T. (2005). Irradiance models. Adv. Space Res., 35, 376383.

Solanki, S. K., Krivova, N. A., \& Haigh, J. D. (2013). Solar Irradiance Variability and Climate. Annu. Rev. Astron. Astrophys.

Soński, M. (2011). Bayesian neural networks and gaussian processes in identification of concrete properties. Computer Assisted Mechanics and Engineering Sciences, 18, 291-302. 
Srivastava, N., Hinton, G., Krizhevsky, A., Sutskever, I., \& Salakhutdinov, R. (2014). Dropout: A simple way to prevent neural networks from overftting. Journal of Machine Learning Research, 15, 1929-1958.

Svozil, D., KvasniEka, V., \& Pospichal, J. (1997). Tutorial: Introduction to multi-layer feed-forward neural networks. Chemometrics and Intelligent Laboratory Systems, 39, 43-62.

de Toma, G., White, O. R., \& Chapman, G. A. (2004). Solar cycle 23: An anomalous cycle? Astrophys J., 609(2), 1140-1152.

de Toma, G., White, O. R., Chapman, G. A., Walton, S. R., Preminger, D. G., Cookson, A. M., \& Harvey, K. L. (2001). Differences in the sun's radiative output in cycles 22 and 23. Astrophysical Journal, 549, L131L134.

Usoskin, I. G. (2013). A history of solar activity over millennia. Living Rev. Solar Phys., 10.

Vaquero, J. M., Gallago, M. C., Trigo, R. M., Sanchez, F., Cancillo, M. L., \& Garcia, J. A. (2006). A new reconstruction of total solar irradiance since 1832. atmósfera, 19, 267-274.

Willson, R., \& Mordvinov, A. (2003). Secular total solar irradiance trend during solar cycles 21-23. Geophys. Res. Lett., 30 .

Yeo, K., Krivova, N., \& Solanki, S. (2014a). Cycle variation in solar irradiance. Space Sci. Rev., 186, 137-167. 
466 Yeo, K., Krivova, N., Solanki, S., \& Glassmeier, K. H. (2014b). Reconstruc467 tion of total and spectral irradiance from 1974 to 2013 based on kpvt, 468 soho/mdi, and sdo/hmi observations. $A \mathscr{E} A, 570, \mathrm{~A} 85$. 